\title{
RESULTADOS PRELIMINARES EN LOS ESTUDIOS DE IMPLANTACIÓN DE ENERGÍAS RENOVABLES EN EL REGADÍO DEL PROYECTO I+D+i OPTIREG: GESTIÓN EFICIENTE DE REGADÍOS
}

\author{
Fernández, K. 1, Ortega, A. 2, Carpio, F.3, Mayordomo, A 4, Martinez, M.A.5, Iglesias, S.6
}

1 Ingeniero Agrónomo, responsable técnico de proyecto OPTIREG1316, Gerencia de Ingeniería y Edificación (Tragsatec), Julián Camarillo 6, 28037 Madrid, kfg@tragsa.es 2 Ingeniero Industrial en proyecto OPTIREG1316, Gerencia de Ingeniería y Edificación (Tragsatec), Julián Camarillo 6, 28037 Madrid, aortega3@tragsa.es

3 Ingeniero Industrial en proyecto OPTIREG1316, Gerencia de Ingeniería y Edificación (Tragsatec), Julián Camarillo 6, 28037 Madrid, fcarpio@tragsa.es

4Ingeniero Agrónomo e Ingeniero de Montes en proyecto OPTIREG1316, Gerencia de Ingeniería y Edificación (Tragsatec), Julián Camarillo 6, 28037 Madrid, amm@tragsa.es 5Ingeniero Técnico Industrial en proyecto OPTIREG1316, Gerencia de Ingeniería y Edificación (Tragsatec), Julián Camarillo 6, 28037 Madrid, mmes@tragsa.es 6 Ingeniero Agrónomo. Master in International Trade. Responsable de apoyo y supervisión de proyecto OPTIREG1316. Subdirección de I+D+i (Tragsa), Conde Peñalver 84, Madrid, siglesia@tragsa.es

\section{Resumen}

OPTIREG, tiene el objetivo de alcanzar nuevas posibilidades de ahorro a partir de una buena gestión en toda la cadena de valor. Para ello trabaja en cuatro bloques interrelacionados: 1) implantación de energías renovables, 2) mercados eléctricos y compraventa de energía, 3) eficiencia hídrica y 4) eficiencia energética.

En el presente Trabajo Técnico se presentan los resultados obtenidos, hasta el momento, en el bloque 1) implantación de energías renovables, mediante el cual se busca una disminución del coste energético del regadío, así como una mayor independencia de los proveedores de electricidad. Se ha investigado el uso y rentabilidad de la energía fotovoltaica, eólica y minihidráulica para su integración en una red de regadío actual.

\section{1.- Introducción.}

La modernización de los regadíos, pasando del riego por gravedad al riego presurizado, trae aparejado un aumento considerable del consumo de energía eléctrica. Esto, sumado al escenario de precios crecientes de este tipo de energía, plantea el estudio de incorporar innovaciones tecnológicas en las zonas regables, tanto actuales como futuras, para conseguir zonas regables eficientes desde el punto de vista energético y económico.

A través del desarrollo del proyecto de $1+D+i$ "Gestión Eficiente de regadíos" (OPTIREG1316) que inició su actividad a mediados del año 2013, el Grupo Tragsa apuesta por dar soluciones al problema energético de las zonas regables. Una óptima administración 
en las zonas regables actuales y futuras permite una mayor eficiencia hidroenergética y por consiguiente una mayor rentabilidad económica en la agricultura de regadío.

La disminución del coste energético debe abordarse mediante una estrategia global: disminuyendo la potencia y la energía consumidas, pero también disminuyendo el coste de las mismas. Lo primero se puede conseguir mediante la mejora de la eficiencia energética de las instalaciones, así como con una programación de riegos óptima. Lo segundo mediante una optimización de la compra de la misma, pero también a través de la correcta instalación de un generador de electricidad mediante energías renovables, el cual sea capaz de ofrecer una electricidad más barata que la adquirida, procedente de la red.

\section{2.- Materiales y Métodos}

El marco regulatorio del sector eléctrico es complejo, debido principalmente a que depende de un gran número de reales decretos y órdenes ministeriales parcialmente derogados, por lo que se ha estudiado la normativa y su evolución desde la liberación del sector en 1997. Las actuales limitaciones que ofrece la normativa a las instalaciones productoras de electricidad mediante energías renovables, junto con la incertidumbre de la evolución de la misma han llevado a cabo la paralización del sector en el país durante los últimos dos años. Las posibilidades técnicas de conexión entre el generador eléctrico y la bomba de agua pasan por una instalación de autoconsumo o por una aislada (también conocida como en isla). Ambas posibilidades se permiten en la actual normativa, sin embargo es mucho más sencilla y previsiblemente estable en el tiempo, la normativa referida a una instalación aislada, ya que en este caso al no estar conectada a la red nacional de electricidad no está sujeta a cambios imprevisibles.

La opción del autoconsumo es muy interesante desde el punto de vista técnico, no sólo porque proporciona una mayor modularidad y seguridad al regante, ya que el $100 \%$ de su instalación sigue conectada a la red convencional, sino también por:

- Producir electricidad durante todo el año, una instalación de autoconsumo convencional permite aprovechar la electricidad generada fuera de la campaña de riego al poderla verter en la red, lo cual acortaría el tiempo de amortización de la instalación. Sin embargo la normativa actual no contempla el balance cero, es decir, compensar dicha energía vertida a la red durante el invierno por aquella que faltará y se consumirá de la red en verano, durante la campaña de riego. Actualmente se puede vender la electricidad excedentaria producida, pero esto conlleva unas importantes complicaciones administrativas y legales, además de ponerse en duda la rentabilidad económica. Vender el excedente de electricidad, tiene en la actualidad las siguientes limitaciones: se cobra precio de mayorista en la venta y se paga precio de minorista en la compra, es obligatorio darse de alta como receptor de IVA y de impuestos especiales, si la potencia de la instalación es mayor de $100 \mathrm{KW}$ la normativa te considera productor de electricidad con la consiguiente complicación administrativa y legal (depósito de aval, etc...).

- Instalaciones de bombeo de mucha potencia (del orden de miles de kilovatios), en las que desconectar una bomba implicaría una instalación de potencia renovable excesiva, ya sea por limitación de la tecnología, superficie, precio...

Por otro lado, la evolución de las tarifas eléctricas en los últimos años, con una subida del término de potencia y una bajada en el de energía disminuyen la rentabilidad de este tipo de 
instalación, ya que es el término de potencia el que no se puede eliminar de la factura eléctrica con estas instalaciones.
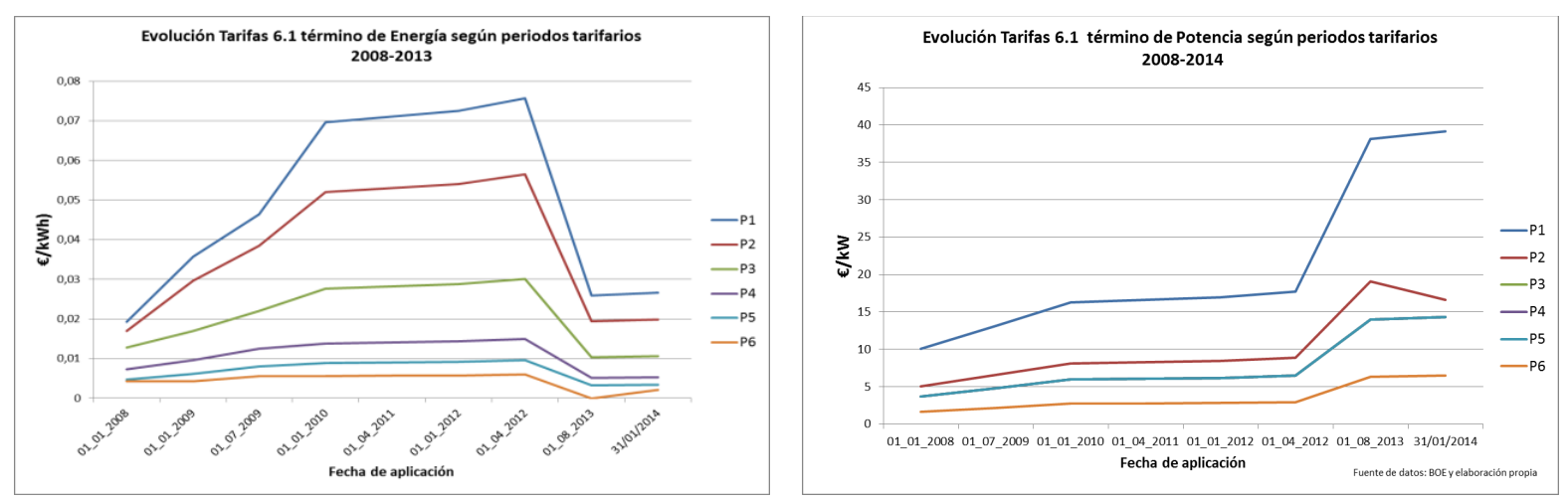

Figura 1. Evolución de la tarifa 6.1

La inversión en una instalación renovable es una inversión a largo plazo, entre 15 y 30 años según tecnología, por lo que la estabilidad jurídica y financiera es importante. Por ello, de momento el proyecto OPTIREG trabajará con instalaciones aisladas de la red.

Las tres fuentes renovables planteadas para producir la electricidad necesaria para el bombeo de agua son: fotovoltaica, eólica y minihidráulica. Cada una de ellas con distintas características que se repasan a continuación.

\section{Fotovoltaica}

Se trata de una tecnología modular, por lo que podemos ir aumentando la potencia instalada $y$, por tanto nuestra inversión, de forma escalonada en el tiempo sin incurrir en costes mayores. El recurso solar está medido en todo el territorio nacional y es previsible en el tiempo y espacio. La evolución de la irradiación recibida en un emplazamiento a lo largo de un año sigue una curva similar a la del consumo energético del regadío, lo que hace de la solar una fuente de energía idónea para el bombeo. Una instalación de bombeo solar crea poco impacto medioambiental, sin embargo en potencias altas como las necesarias para una comunidad de regantes, la extensión necesaria para los paneles puede llegar a ser importante, unos $2.500 \mathrm{~m}^{2}$ para una instalación de $100 \mathrm{~kW}$. La fotovoltaica aislada es una tecnología madura en el país, por lo que aunque su aplicación en el bombeo de alta potencia es reciente, resulta sencillo y barato encontrar empresas de mantenimiento.

\section{Eólica}

La eólica es una tecnología no modular, y que además ofrece mejores precios unitarios a mayor potencia, lo que hace que debamos dimensionar la potencia del aerogenerador para toda la vida de la instalación desde el principio y de manera muy precisa. El viento es un recurso mucho más variable que el sol, y mucho más difícil de medir. Para una correcta caracterización del viento en una ubicación sería necesaria una campaña de mediciones a la altura del eje del generador durante al menos un año, lo cual implicaría un coste y un tiempo demasiado elevados para una instalación de este tipo. El viento en una ubicación se caracteriza mediante la función de Weibull, distribución de probabilidad de la velocidad del 
viento en la ubicación. Existen bases de datos para cualquier punto del país basadas en modelos numéricos apoyados en datos de estaciones meteorológicas, este tipo de base de datos son usadas por los pequeños instaladores de eólica. Sin embargo, en la mayoría de casos no existirá una ubicación cercana al punto de consumo con una cantidad de viento suficiente como para hacer rentable la instalación. Aún en los puntos de consumo donde sí se disponga de buenos vientos para la generación de electricidad, lo más probable es que en unas decenas de kilómetros exista una ubicación con suficiente viento como para que sea rentable la construcción de una pequeña línea de transporte de electricidad con sus correspondientes transformadores.

Tanto la energía eólica como la minihidráulica tienen el problema, al trabajar en aislada, de la excitación del generador eléctrico utilizado, ya que normalmente se utilizan generadores asíncronos en estas tecnologías. En estos casos la inversión se encarecería y complicaría ligeramente en comparación con una instalación conectada a la red.

\section{Minihidráulica}

El recurso hidráulico para la generación de electricidad en una comunidad de regantes es muy concreto y limitado. A diferencia de las otras tecnologías, en este caso el recurso renovable aprovechable para la generación de electricidad no depende de su ubicación, sino de su red de riego, lo cual hace que:

- Dicho recurso sea perfectamente conocido y predecible al $100 \%$, por lo que podremos saber en todo momento la cantidad de energía que se generará.

- Sea difícil de calcular sin conocer con detalle la red de riego.

Su instalación no genera ningún tipo de impacto en el medio, ya que se integra en la red de agua. Conlleva un mayor coste de inversión inicial, pero si se dispone de un salto de presión de tamaño suficiente el coste de la energía obtenida es realmente competitivo.

Para el desarrollo del proyecto se ha llevado a cabo un estudio básico sobre la viabilidad técnica y económica en una veintena de comunidades de regantes interesadas. Entre estas comunidades se han elegido, siguiendo criterios tanto técnicos como económicos y organizativos, aquellas que servirán de piloto en el proyecto. En dichas comunidades piloto se llevará a cabo un estudio detallado de las posibilidades técnicas reales de implantación de energías renovables en las actuales instalaciones de regadío. Aquellas comunidades que decidan llevar a cabo las instalaciones propuestas por OPTIREG recibirán el asesoramiento necesario para llevar a cabo con seguridad y tranquilidad su inversión.

\section{3.- Resultados y Discusión}

La integración de energías renovables en aislado, en las instalaciones actuales de regadío en España conlleva una serie de necesidades y restricciones en las redes (hidráulica y eléctrica) mínimas, tanto desde el punto de vista técnico como administrativo. La mayoría de instalaciones cumplen estas necesidades y restricciones sin mayor problema. El caso de la minihidráulica es la excepción, ya que para la producción de electricidad con un rendimiento y unos costes competitivos se hace necesario un salto de presión importante ubicado cerca del punto de consumo. 
El principal parámetro que afecta a la rentabilidad de una instalación de este tipo es la cantidad de recurso renovable disponible. Tanto en fotovoltaica como en eólica éste depende de la ubicación, no es así el caso de la minihidráulica, que como se ha explicado depende únicamente de la red de riego. El recurso solar es predecible y varía poco en tiempo y espacio, sin embargo el eólico varía en gran medida tanto en tiempo como en espacio, lo que dificulta su caracterización.

Definiendo el factor de capacidad como la cantidad de horas al año (totales o relativas) que tendría que haber estado funcionando una instalación generadora de electricidad a su potencia nominal para generar la misma cantidad de energía que en condiciones reales podemos afirmar que:

- El factor de capacidad de una de una instalación fotovoltaica:

- Es predecible en gran medida.

- Varía poco en todo el territorio español.

- Es afectado principalmente por la irradiación local y por el calendario de riego.

- El factor de capacidad de una instalación eólica:

- Es difícil de predecir con precisión.

- Varía mucho en decenas de kilómetros.

- Es afectado principalmente por la distribución de Weibull en la ubicación del aerogenerador y por el calendario de riego.

Puesto que en una instalación de renovables la fuente de energía es gratuita, y por lo tanto los costes variables prácticamente nulos, el factor de capacidad influirá en gran medida en la rentabilidad de la instalación. A mayor factor de capacidad, mayor cantidad de energía eléctrica generada por la instalación, y por lo tanto se obtiene mayor rentabilidad, ya que los costes se mantienen prácticamente constantes.

A modo resumen se muestra la siguiente tabla para comparar tecnologías:

\begin{tabular}{|l|c|c|c|}
\hline \multicolumn{1}{|c|}{ Tecnología } & $€ / \mathrm{KW}$ & $\mathrm{FC}$ & $€ / \mathrm{MW} \cdot \mathrm{h}$ \\
\hline Fotovoltaica & $1300-1700$ & $0,11-0,17$ & 63 \\
\hline Eólica más de $300 \mathrm{KW}$ & 1000 & $<0,34$ & $>32$ \\
\hline Eólica menos de $300 \mathrm{KW}$ & $2000-6000$ & $<0,56$ & $>36$ \\
\hline Minihidráhulica & 3000 & $0,2-0,5$ & 52 \\
\hline
\end{tabular}

*Datos y elaboración propios, precios con IVA incluido

En la columna $€ / \mathrm{KW}$ se indica el precio aproximado del coste de la instalación, todo incluido. En el caso de la Eólica de menos de $300 \mathrm{KW}$ se indica un margen de precios medios amplio, ya que varía en gran medida; disparándose los costes por KW en instalaciones de menos de $100 \mathrm{KW}$.

En la columna FC se muestran unos rangos de factores de capacidad tipo. En el caso de la fotovoltaica se han considerado instalaciones fijas, ya que la instalación de seguidores no es 
rentable económicamente. En el caso de eólica se ha indicado el factor de capacidad máximo, ya que al variar tanto es imposible dar un valor medio, este factor de capacidad máximo es posible en muy pocas ubicaciones, por lo que hay que tener en cuenta que el precio indicado de la energía no es aplicable en la mayoría de casos. El factor de capacidad de una instalación minihidráulica dependerá principalmente de las horas de riego, por lo que en realidad se podrá tratar como un porcentaje de ahorro directo sobre la energía que se consume procedente de la red.

La rentabilidad económica de una instalación de este tipo viene dada por el ahorro producido al disminuir el consumo de electricidad, tanto de energía como de potencia. El uso de energías renovables implica una fuerte inversión inicial, (con o sin préstamo externo) a cambio de una producción de electricidad "prácticamente gratuita" durante toda la vida de la instalación, por lo que el ahorro real ofrecido por una instalación renovable depende del precio de adquisición de la electricidad por parte del consumidor. Este precio varía en gran medida de un consumidor a otro y depende de muchos factores: optimización del contrato y relación potencia contratada - energía consumida, optimización de periodos de facturación, tipo de contrato (precio fijo, indexado...), cantidad de compra total (agrupaciones de cc.rr. en juntas de compras).

La optimización en la compra de electricidad por parte de una cc.rr. es compleja, por lo que es otra línea de trabajo de OPTIREG, cuyos resultados se presentaron parcialmente en el XXXII Congreso Nacional de Riegos. En los últimos dos años el precio a tarifa ha variado enormemente, al sufrir éstas grandes cambios. Unos precios óptimos obtenidos de forma sencilla en los últimos años por las cc.rr. son aquellos conseguidos mediante su agrupación en juntas de compras, cuyo precio estimado en $110 € / \mathrm{MW} \cdot \mathrm{h}$ podemos usar como parámetro de base para el cálculo de la rentabilidad de una instalación de energías renovables.

Según Eurostat, la electricidad ha aumentado en los últimos 15 años aproximadamente un $100 \%$ para consumidores industriales, lo que implica una subida superior al $6 \%$ anual. Para los cálculos de rentabilidad económica hemos considerado un precio constante de la electricidad, ya que esta tendencia podría llegar a revertirse si se dan las condiciones necesarias, principalmente un cambio en la normativa española actual y uso de fuentes de electricidad renovables, no dependientes del precio del petróleo.

Es de esperar que la rentabilidad mostrada en este estudio sea menor que la real final, sin embargo se ha preferido considerar un escenario conservador.

En el caso de bombeo solar la rentabilidad calculada varía desde el $6 \%$ anual en Segovia, regando de marzo a octubre y desperdiciando la electricidad generada fuera de la campaña de riego, lo que ofrece un plazo de recuperación de la inversión de 14 años para el agricultor. Si se pudiera aprovechar la irradiación recibida de noviembre a febrero este periodo se reduciría hasta los 9 años. En Almería, donde la irradiación es mayor y se riega durante todo el año la rentabilidad aumenta hasta el $10 \%$, reduciendo el plazo de recuperación hasta los cuatro años.

En el caso del bombeo eólico la rentabilidad varía enormemente de una ubicación a otra, siendo imposible dar resultados a nivel nacional. Debido a la mayor incertidumbre que conlleva una instalación eólica de este tipo respecto a una fotovoltaica, su recomendación 
será positiva únicamente en el caso de obtener una rentabilidad superior al $6 \%$ en el estudio previo.

De manera similar, con la minihidráulica no es posible ofrecer estimaciones de rentabilidad a nivel nacional, siendo necesario llevar a cabo la potencialidad de la instalación en cada red de riego individualizadamente.

\section{4.- Conclusiones y Recomendaciones}

En los últimos años se ha producido una fuerte subida en los costes eléctricos, tanto para grandes consumidores como para consumidores domésticos. Esto, unido al aumento en el consumo como consecuencia de las modernizaciones en los regadíos, ha provocado un gran aumento de costes energéticos para las cc.rr. que debe ser abordado con varias soluciones, como es el uso de ee.rr..

Tanto la fotovoltaica como la eólica o la minihidráulica están extendidas en España. Se trata de tecnologías maduras, que sin embargo, no han sido aplicadas aún de forma mayoritaria en el regadío de alta potencia.

La tecnología fotovoltaica es ideal para el bombeo de agua para regadío, ya que cuando más se necesita más disponibilidad ofrece, además de funcionar sin ningún tipo de problema y con un mantenimiento prácticamente nulo en régimen aislado.

La eólica resulta más compleja que la fotovoltaica y rentable en menos localizaciones, sin embargo, tiene una gran potencialidad en algunas zonas.

La minihidráulica depende totalmente de la red de riego y debe ser estudiada más a fondo en cada caso. Puede llegar a ofrecer una electricidad realmente barata y el mantenimiento que necesita es muy básico.

En general, la principal restricción en el uso de renovables para el bombeo de agua en el regadío es la cantidad de recurso renovable disponible, por lo que su aplicación puede llegar a extenderse de forma mayoritaria en el sector, sobre todo la fotovoltaica. 